\title{
Compressive Sensing in Microscopy: a Tutorial
}

Andrew Stevens ${ }^{1,2}$, Hao Yang ${ }^{3}$, Libor Kovarik $^{1}$, Xin Yuan ${ }^{4}$, Quentin Ramasse ${ }^{5}$, Patricia Abellan ${ }^{5}$, Yunchen $\mathrm{Pu}^{2}$, Lawrence Carin ${ }^{2}$, and Nigel D. Browning ${ }^{1}$

1. Pacific Northwest National Laboratory, Richland USA

2. Duke University, ECE, Durham USA

3. Lawrence Berkeley National Laboratory, Berkeley USA

4. Bell Laboratories, Murray Hill USA

5. SuperSTEM, STFC Daresbury Laboratories, Warrington UK

Currently many types of microscopy are limited, in terms of spatial and temporal resolution, by hardware (e.g., camera framerate, data transfer rate, data storage capacity). The obvious approach to solve the resolution problem is to develop better hardware. An alternative solution, which additionally benefits from improved hardware, is to apply compressive sensing (CS) [1]. CS approaches have been shown to reduce dose by as much as $90 \%$ in electron microscopy $[2,3,4]$. Optical imaging and microscopy have also seen substantial benefits $[5,6,7,8,9,10,11,12,13]$.

This tutorial will briefly introduce the principles of CS. Primarily, we will focus on the setup and modifications necessary for applying CS to a few different types of microscopy and spectroscopy (e.g., STEM [2], EELS, TEM-video [3], optical-video [7]). We will show results from these compressive microscopy approaches. Moreover, an approach for detecting CS reconstruction errors (i.e., errors introduced by the image processing algorithm) will be discussed.

Two examples are shown in Figure 1. The first example shows a 10:1 compression of video transmission electron (TEM) microscopy data. The second example shows the reconstruction of a scanning TEM (STEM) image using only $20 \%$ of the pixels [14].

\section{References:}

[1] RG Baraniuk. IEEE signal processing magazine 24(4).

[2] A Stevens, H Yang, L Carin et al. Microscopy 63(1), (2014), pp. 41.

[3] A Stevens, L Kovarik, P Abellan et al. Advanced Structural and Chemical Imaging 1(1), (2015), pp. 1.

[4] A Stevens, L Kovarik, P Abellan et al. Microscopy and Microanalysis 21(S3), (2015), pp. 1583.

[5] M Zhou, H Chen, J Paisley et al. Image Processing, IEEE Transactions on 21(1), (2012), pp. 130.

[6] Z Xing, M Zhou, A Castrodad et al. SIAM Journal on Imaging Sciences 5(1), (2012), pp. 33.

[7] X Yuan and S Pang. Biomedical Optics Express 7(3), (2016), pp. 746.

[8] Y Pu, X Yuan and L Carin. arXiv:14126039.

[9] Y Pu, X Yuan, A Stevens et al. In AISTATS 2016.

[10] P Llull, X Liao, X Yuan et al. Optics Express 21(9), (2013), pp. 10526.

[11] X Yuan, J Yang, P Llull et al. In ICIP 2013 (IEEE).

[12] J Yang, X Yuan, X Liao et al. Image Processing, IEEE Transactions on 23(11), (2014), pp. 4863.

[13] X Yuan, P Llull, X Liao et al. In CVPR 2014 (IEEE).

[14] This work was supported in part by US DOE Grant No. DE-FG02-03ER46057. This research is also part of the Chemical Imaging Initiative conducted under the LDRD Program at PNNL and used EMSL, a national scientific user facility sponsored by the DOE Office of Bio \& Env. Research at PNNL. PNNL is a multi-program national lab operated by Battelle Memorial Institute under Contract DE-AC05-76RL01830 for the US DOE. 

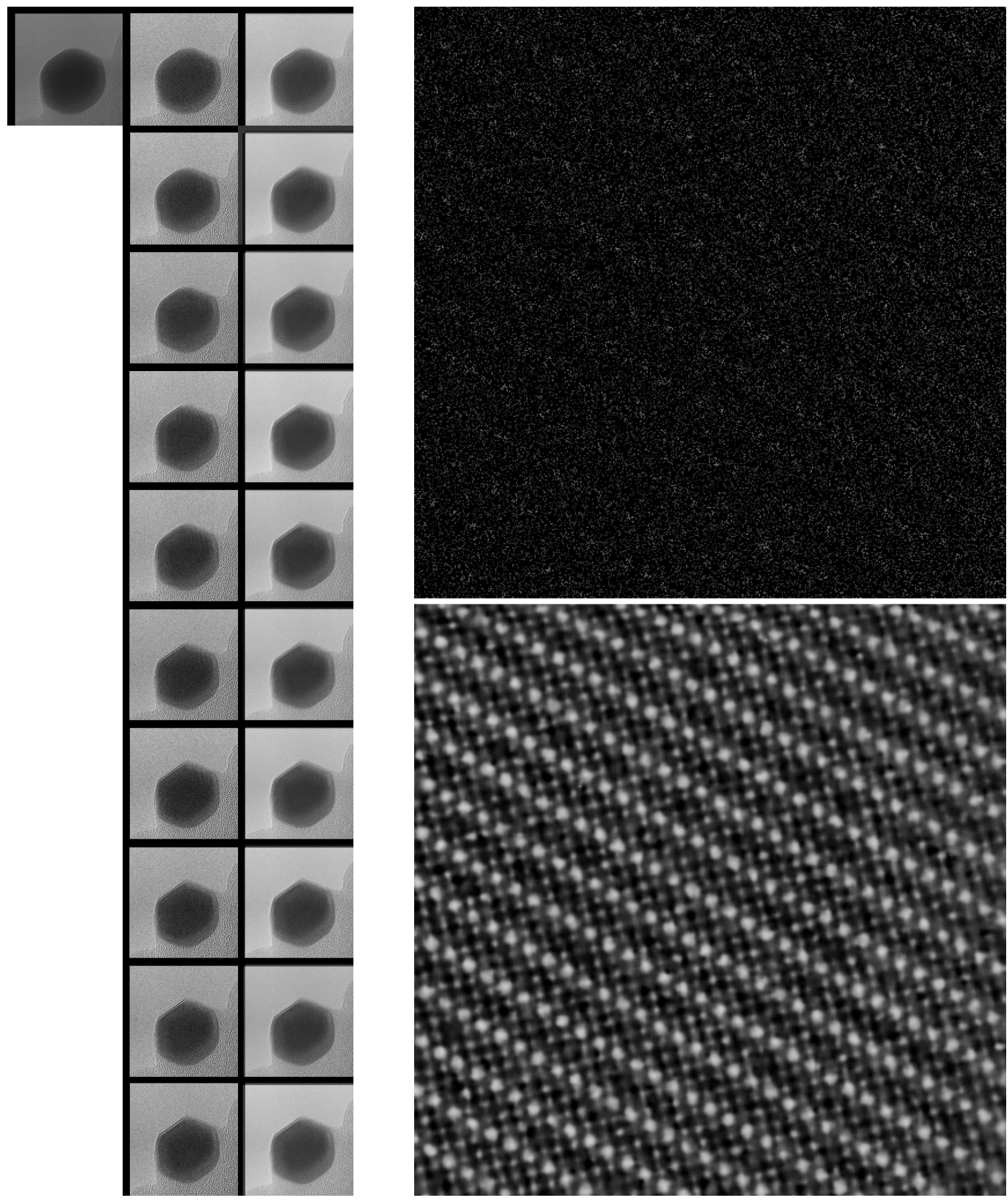

Figure 1: Left: An illustration of CS-TEM with 10 video frames compressed into 1 (simulated). The top left image shows the compressed frame, the middle column of images shows the reconstructed frames, and the right column shows the original frames. Right: An example of CS-STEM with $80 \%$ of the pixels missing at random (experimental). The top image is the acquired data, and the bottom image is the CS reconstruction. 